\title{
A Geometrical Theorem about the Static Equilibrium of a Common-point-force System and its Application
}

\author{
Guo-quan Zhou \\ Department of Physics, Wuhan University, Wuhan, Hubei, China \\ Email: zgq@whu.edu.cn
}

Received 2013

\begin{abstract}
A geometrical theorem for the static equilibrium of a common-point-force system has been proven by means of virtual-work principle: The equilibrium point of a common-point force system has a minimal weighted distance summation to every fixed point arbitrarily given on each force line with a weighing factor proportional to corresponding force value. Especially the mechanical simulating technique for its inverse problem has been realized by means of pulley block. The conclusions for the inverse problem derived from mechanic method are in accordance with that given by the pure mathematical method, and the self-consistence of the theorem and its inverse problem has been demonstrated. Some application examples in engineering, economy and mathematics have been discussed, especially the possible application in the research of molecular structure, has also been predicted.
\end{abstract}

Keywords: Spatial Common-point-force System; Static Equilibrium; Minimum Theorem; Principle of Virtual Work; Depot Problem; Transportation

\section{Arising of the Problem and its Mathematical Model}

The so-called depot problem [1-5], one of the edging subject in the field of transportation science and modern architectural economy, is related directly to the economic efficiency and the industrial and commercial interests. The suitably chosen "depot" can save much man power and working time, decrease unnecessary loss of material resources. Recent years, with the fast development of mathematics, computer technology and operational research, some methods have been found to solve the multi-depot vehicle routing problem, such as the Integer Programming Model combined with the Hybrid Genetic Algorithm, the Nearest Neighbor Heuristic Method, and the Tabu Search Method based on Adaptive Memory Principle[1-5], and so on. One of the basic problems is just like the following example.

Provided $\mathrm{P}_{1}, \mathrm{P}_{2}, \cdots, \mathrm{P}_{N}$ are $N$ disperse working sites for vehicle transportation, the possible location of the main traffic station $\mathrm{P}$ has mean $n_{i}$ times of to-and-fro transportation between $\mathrm{P}$ and $\mathrm{P}_{i}(i=1,2, \cdots \cdots, N)$ each month, then which location $\mathrm{P}$ can have a minimal weighted distance summation to every working site and minimize the total fuel consumption?

This question can be expressed as a mathematical question: to look for a point $P$ which can minimize the double weighted distance summation:

$$
\begin{aligned}
S & =2 \sum_{i=1}^{N} n_{i} \cdot P P_{i} \\
& =2\left(n_{1} \cdot P P_{1}+n_{2} \cdot P P_{2}+\cdots \cdots+n_{N} \cdot P P_{N}\right)
\end{aligned}
$$

From the viewpoint of fuzzy mathematics, number $n_{i}$ 's---- the weighing factors appeared in above questions are average values with respect to time and needn't to be integers, they can be any positive real numbers.

A solution to such a problem is found to be related to a mechanical model - the static equilibrium of a common-point-force system (CPF system for brevity), which is based on the principle of minimal potential for the static equilibrium of a conservative mechanical system in the gravitational field. Another equivalent method of virtual-work principle under ideal constraint is an alternative solution to the inverse case of above problem. In the following section, we will throw light on such a fact that static equilibrium method of CPF system can solve the problem of least weighted distance summation for both two and three dimensional cases. It can also be used to deal with cases involved in not only the straight path but also the blocked or curved path.

\section{A Geometrical Theorem for the Balanced CPF System}

Based on the principle of virtual work, a geometrical theorem for the static equilibrium of a CPF system can 
be easily proved. Then the correlation between the theorem and above problems will be immediately established after a suitable mechanic model has been constructed.

Theorem The equilibrium point of a common-point force system has a minimal summation of weighted distance from every fixed point arbitrarily given on each force line, with a weighing factor proportional to corresponding force value.

The above theorem means, for $N$ balanced forces $\boldsymbol{F}_{i}$ with common point $P$ and given values $n_{i} f$ respectively $(i=1,2, \cdots N$, and $f$ is a common factor with force's dimension) and for $N$ fixed points $P_{i}(i=1,2, \cdots N)$ in each force line respectively, the equilibrium point $P$ of a CPF system must minimize the weighted distance summation $P S=\sum_{i=1}^{N} n_{i} \cdot P P_{i}$, i.e. for any other point $P^{\prime}$,

$$
S^{\prime}=\sum_{i=1}^{N} n_{i} \cdot P^{\prime} P_{i} \geq S_{\min }=\sum_{i=1}^{N} n_{i} \cdot P P_{i}
$$

where $n_{i}(i=1,2, \cdots N)$ satisfy following relation:

$$
\left|\boldsymbol{F}_{1}\right|:\left|\boldsymbol{F}_{2}\right|: \cdots \cdots:\left|\boldsymbol{F}_{N}\right|=n_{1}: n_{2}: \cdots \cdots: n_{N}
$$

By means of virtual-work principle about stable equilibrium of a multi-force system, the above theorem can be easily proven as follows.

For an arbitrary virtual displacement $\delta \boldsymbol{r}$ (from $\left.P \rightarrow P_{0}\right)$ of the equilibrium position $P$ and $\delta \boldsymbol{r}_{i}$ of each fixed point $P_{i}(i=1,2, \cdots N)$, suppose $\overrightarrow{P P_{i}}=\boldsymbol{r}_{i}$, the virtual work of the resultant force $\boldsymbol{F}=\sum_{i=1}^{N} \boldsymbol{F}_{i}$ is zero under ideal constraint, that is

$$
\boldsymbol{F} \cdot \boldsymbol{\delta} \boldsymbol{r}=\sum_{i=1}^{N} \boldsymbol{F}_{i} \cdot \boldsymbol{\delta} \boldsymbol{r}=0
$$

or

$$
\sum_{i=1}^{N} \boldsymbol{F}_{i} \cdot \boldsymbol{\delta} \boldsymbol{r}_{i}=0
$$

which is duo to following fact

$$
\boldsymbol{\delta} \boldsymbol{r}=\boldsymbol{\delta} \boldsymbol{r}_{i}(i=1,2, \cdots \cdots, N)
$$

On the same time, forces $\vec{F}_{i}(i=1,2, \cdots N)$ are all centric forces that respectively direct at fixed point $P_{i}$ $(i=1,2, \cdots N)$, so we have

$$
\boldsymbol{F}_{i}=\left(n_{i} f\right) \frac{\boldsymbol{r}_{i}}{r_{i}}
$$

where $\left|\boldsymbol{F}_{i}\right|=n_{i} f, f$ is the common factor with dimension of force and $n_{i}$ the dimensionless positive real constant; $\frac{r_{i}}{r_{i}}$ is the unit vector along with $\overrightarrow{P P_{i}}$ or the direction of force $\vec{F}_{i}$ : according to the property of centric force[7-9], its work can be express as

$$
\boldsymbol{F}_{i} \cdot \boldsymbol{\delta} \boldsymbol{r}_{i}=n_{i} f\left(-\delta r_{i}\right)
$$

Then the condition (2) can be written as:

$$
\begin{gathered}
\sum_{i=1}^{N} n_{i} f\left(-\delta r_{i}\right)=0 \\
\delta \sum_{i=1}^{N} n_{i} r_{i}=0
\end{gathered}
$$

This is just the necessary and in fact also the sufficient condition for weighted distance summation

$$
\sum_{i=1}^{N} n_{i} r_{i}=\sum_{i=1}^{N} n_{i} \cdot\left|P P_{i}\right|
$$

to attain its minimum. Then the above theorem get verified.

For the special case that $n_{i}>\sum_{j=1, j \neq i}^{N} n_{j}$ (a sufficient but not necessary condition), the CPF system can't attain equilibrium state by itself, then point $P$ will move to certain a point $P_{i}$ and attain equilibrium by means of forced constraint reaction at $P_{i}$. Thus, point $P_{i}$ is just the wanted point $P$ to minimize the sum $\sum_{i=1}^{N} n_{i} \cdot P P_{i}$.

The theorem (2) is actually the mathematical criterion for the static equilibrium of the CPF system. On the other hand, this geometry theorem also clearly reflects the geometric property of the CPF system having attained equilibrium state, and what is more, reflects the spatial property of the concerted fields of forces.

On the other hand, a stable static equilibrium of the CPF system corresponds to the case of the minimal sum, and an unstable static equilibrium corresponds to the case of the maximal sum.

\section{A Mechanical Model and Solution to the Inverse Problem}

There had ever been some discussion in history about the so-called depot problem or factory-location problem similar to question in section 1 . It is easily found to be the inverse problem of the above theorem, and can be solved by mechanic technique. Reference [6] had given two mechanic models to solve such a geometrical problem in the two-dimensional case, one is the model of

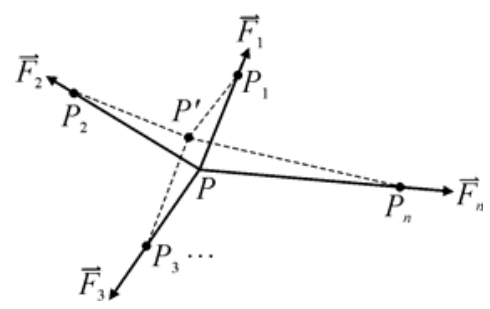

Figure 1. The balanced common-point-force system. 
surface tension of liquid cake films, the other is the model of ropes with suspended weights passing through smooth holes on a frictionless table. But in three-dimensional case, both models are invalid. In order to find a feasible technique to solve the problem in the three-dimensional case, we can always proportionately minify the real distribution of $\mathrm{N}$ given point $P_{1}, P_{2}, \cdots P_{N}$ to a model of miniature distribution $P_{1}^{\prime}, P_{2}^{\prime}, \cdots P_{N}^{\prime}$ in laboratory, shown as Figure 2, then install $N$ frictionless fixed pulleys at each point $N P_{i}^{\prime}(i=1,2, \cdots N)$. Each rope with length of $L$ is connected respectively to a suspended weight $G_{i}^{\prime}$ of mass $n_{i}$ at one end and to the common point $P$ at the other end, and passes by the fixed pulley at $P_{i}^{\prime}$ respectively. Note that each fixed pulley has a definite position and adjustable free axis. Obviously the whole $\mathrm{CPF}$ system is a pure gravitation-doing-work system under ideal constraint, i.e. a conservative system. When the system attains static equilibrium, the whole system will possess the minimal total gravitation potential energy with respect to any given horizontal plane $\alpha$, which is chosen to be the zero plane of potential energy (shown as Figure 2). If each of the point of $P_{1}^{\prime}, P_{2}^{\prime}, \cdots P_{N}^{\prime}$ has the height of $h_{1}, h_{2}, \cdots h_{N}$ respectively with respect to plane $\alpha$, then each particle $G_{i}^{\prime}$ has its gravitation potential energy of $n_{i} g\left(h_{i}-P_{i}^{\prime} G_{i}^{\prime}\right)$ respectively $(i=1,2, \cdots n)$. Then the total gravitation potential energy $\sum_{i=1}^{N} n_{i} g\left(h_{i}-P_{i}^{\prime} G_{i}^{\prime}\right)$ must get the minimal for the equilibrium state, and $\sum_{i=1}^{N} n_{i} g \cdot P_{i} G_{i}^{\prime}$ must attain maximum because $\sum_{i=1}^{N} n_{i} g \cdot h_{i}$ is a constant. Duo to $P_{i}^{\prime} G_{i}^{\prime}=L-P P_{i}^{\prime}$, then $\sum_{i=1}^{N} n_{i}\left(L-P P_{i}^{\prime}\right)$ must be the maximal, and $\sum_{i=1}^{N} n_{i} \cdot P P_{i}^{\prime}$ must attain its minimum.

The condition is that the whole common-point-force system attains its static equilibrium. And the equilibrium position $P$ is just the wanted point to make the weighted distance summation $\sum_{i=1}^{N} n_{i} \cdot P P_{i}^{\prime}$ get its minimum.

It should be pointed out once more that the weighted distance summation may attain its minimum at the boundary point such as $P_{i}^{\prime}(i=1,2, \cdots N)$ - when the equilibrium state can only be attained by means of forced constraint reaction at $P_{i}^{\prime}$, then the point $P_{i}^{\prime}$ is just the wanted point satisfying the minimum condition. Here, we give a sufficient but not necessary condition, that is:

When $n_{i} \geq \sum_{j=1, j \neq i}^{N} n_{j}$, point $P_{i}^{\prime}$ is just the wanted point that makes the summation $S=\sum_{i=1}^{N} n_{i} \cdot P P_{i}^{\prime}$ the minimal.

The condition is that the whole common-point-force system attains its static equilibrium. And the equilibrium position $P$ is just the wanted point to make the weighted distance summation $\sum_{i=1}^{N} n_{i} \cdot P P_{i}^{\prime}$ get its minimum.

It should be pointed out once more that the weighted distance summation may attain its minimum at the boundary point such as $P_{i}^{\prime}(i=1,2, \cdots N)$ - when the equilibrium state can only be attained by means of forced constraint reaction at $P_{i}^{\prime}$, then the point $P_{i}^{\prime}$ is just the wanted point satisfying the minimum condition. Here, we give a sufficient but not necessary condition, that is:

When $n_{i} \geq \sum_{j=1, j \neq i}^{N} n_{j}$, point $P_{i}^{\prime}$ is just the wanted point that makes the summation $S=\sum_{i=1}^{N} n_{i} \cdot P P_{i}^{\prime}$ the minimal.

For many years economists had ever misinterpreted this mechanical analogue of the depot problem in the 2-dimensional case, and mistakenly thought the center of mass $P_{c}$ to be the wanted point to make the weighted distance summation minimal. Actually, $P_{c}$, the center of mass, leads to the minimal weighted square distance summation and not the minimal weighted distance summation.

\section{The Concrete Conclusion for the Three-Force System}

For the simple case such as 3-CPF or 4-CPF system, the above mechanical model will directly lead to some interesting and valuable conclusions.

In field of geometry, we can prove following conclusion by means of pure mathematical method:

For any triangle $\Delta P_{1} P_{2} P_{3}$ with its three inner angles not bigger than $120^{\circ}$, there must be a point $\mathrm{P}$ within the triangle to minimize the distance summation

$$
P P_{1}+P P_{2}+P P_{3}
$$

when and only when

$$
\angle P_{1} P P_{2}=\angle P_{2} P P_{3}=\angle P_{3} P P_{1}=2 \pi / 3 .
$$



Figure 2. The mechanical model. 
The same conclusion can be drawn by means of static equilibrium of common-point system, and what is more, the conclusion can be extended further.

For $N=3,\left|\boldsymbol{F}_{1}\right|:\left|\boldsymbol{F}_{2}\right|:\left|\boldsymbol{F}_{3}\right|=n_{1}: n_{2}: n_{3}$, it is easy to deduce the sufficient and necessary condition for the equilibrium of 3-CPF system, that is

(1), the three forces are coplanar.

(2), following equation must be satisfied:

$$
\frac{\left|\boldsymbol{F}_{1}\right|}{\sin \left(\boldsymbol{F}_{2}, \boldsymbol{F}_{3}\right)}=\frac{\left|\boldsymbol{F}_{2}\right|}{\sin \left(\boldsymbol{F}_{3}, \boldsymbol{F}_{1}\right)}=\frac{\left|\boldsymbol{F}_{3}\right|}{\sin \left(\boldsymbol{F}_{1}, \boldsymbol{F}_{2}\right)}
$$

Expression (10) is just the Lami's theorem [7]. Here $\left(\boldsymbol{F}_{i}, \boldsymbol{F}_{j}\right)$ expresses the angle between two vectors $\boldsymbol{F}_{i}$ and $\boldsymbol{F}_{j}$ (not larger than $\pi$ ).

According the theorem (2), when expression (10) is satisfied, corresponding point $P$ is just the wanted point to make the weighted distance summation

$$
n_{1} \cdot P P_{1}+n_{2} \cdot P P_{2}+n_{3} \cdot P P_{3}
$$

minimal. Then it means

$$
\frac{n_{1}}{\sin \left(\boldsymbol{F}_{2}, \boldsymbol{F}_{3}\right)}=\frac{n_{2}}{\sin \left(\boldsymbol{F}_{3}, \boldsymbol{F}_{1}\right)}=\frac{n_{3}}{\sin \left(\boldsymbol{F}_{1}, \boldsymbol{F}_{2}\right)}
$$

Considering $\left(\vec{F}_{1}, \vec{F}_{2}\right)+\left(\vec{F}_{2}, \vec{F}_{3}\right)+\left(\vec{F}_{3}, \vec{F}_{1}\right)=2 \pi$, we make a transformation

$$
\left\{\begin{array}{l}
\alpha_{1}=\pi-\left(\boldsymbol{F}_{2}, \boldsymbol{F}_{3}\right) \\
\alpha_{2}=\pi-\left(\boldsymbol{F}_{3}, \boldsymbol{F}_{1}\right) \\
\alpha_{3}=\pi-\left(\boldsymbol{F}_{1}, \boldsymbol{F}_{2}\right)
\end{array}\right.
$$

Then $\alpha_{1}+\alpha_{2}+\alpha_{3}=\pi$, and $\alpha_{1}, \alpha_{2}, \alpha_{3}$ can be viewed as the three inner angles of certain a triangle. According to the sine theorem and the cosine theorem, following identity holds

$$
\begin{array}{r}
\cos \alpha_{i}=\frac{\sin ^{2} \alpha_{j}+\sin ^{2} \alpha_{k}-\sin ^{2} \alpha_{i}}{2 \sin \alpha_{j} \cdot \sin \alpha_{k}}=\frac{n_{j}^{2}+n_{k}^{2}-n_{i}^{2}}{2 n_{j} n_{k}} \\
\text { (c.f. (11)) }
\end{array}
$$

Here $i, j, k$ can be permutated in turn within $1,2,3$. Then

$$
\left(\boldsymbol{F}_{j}, \quad \boldsymbol{F}_{k}\right)=\pi-\arccos \frac{n_{j}^{2}+n_{k}^{2}-n_{i}^{2}}{2 n_{\mathrm{j}} n_{k}}
$$

So when the CPF system attains static equilibrium, the open angle of point $P$ with respect to $P_{1} P_{2}, P_{2} P_{3}, P_{3} P_{1}$ respectively can be calculated according to (14).

Specially when $n_{1}=n_{2}=n_{3}=n$ according to (14)

$$
\begin{aligned}
\alpha_{1}=\alpha_{2}= & \alpha_{3}=\frac{\pi}{3} \text {, then } \\
& \left(\boldsymbol{F}_{1}, \boldsymbol{F}_{2}\right)=\left(\boldsymbol{F}_{2}, \boldsymbol{F}_{3}\right)=\left(\boldsymbol{F}_{3}, \boldsymbol{F}_{1}\right)=2 \pi / 3
\end{aligned}
$$

Meanwhile the corresponding point $P$ will make
$S=n\left(P P_{1}+P P_{2}+P P_{3}\right)$ the minimal. This conclusion is just coincident with that given by pure geometrical method. This is not only one of the special application examples of CPF system, but also a convincing geometrical side proof for the above theorem (2) and the mechanical technique. By the way, it is worthy of pointing out, when $n=3$ and $n_{1} \geq n_{2}+n_{3}, \quad P_{1}$ is the needed point that makes $n_{1} \cdot P P_{1}+n_{2} \cdot P P_{2}+n_{3} \cdot P P_{3}$ be the minimal (here $\left.P P_{1}=0\right)$, or $S_{\text {min }}=n_{2} \cdot P_{1} P_{2}+n_{3} \cdot P_{1} P_{3}$.

By means of the same method, we can easily extend our conclusions for case of $n=3$ to the case of $n>3$.

\section{Extension of Application Fields and Some Discussion}

The theorem (2) and the mechanical model given by this paper can be used to deal with both the coplanar CPF case and spatial CPF case. If we replace the pulley block at points of $P_{1}, P_{2} \cdots P_{n}$ by $n$ smooth and frictionless small hole on the surface of a sphere crust, an ideal constraint case, the principle of virtual work and the method of minimal total potential energy are still valid. As a result, many geometric questions on the surface of a sphere can be solved at once. Just as what has been mentioned before, theorem (2) reflects the spatial property of the gravitation fields near to surface of earth. On a large space in the gravitation field, the conclusion must have a little difference from above because of the different expression of gravitation potential energy, and there must be also some spatial distortion effects resulting from the gravitation of the earth.

One of the most interesting applications of the theorem and the mechanical model may be research of molecular structure. A stable molecular structure of multi-atom molecule must obey a geometrical constraint condition. Every chemical bond has definite bond strength and a bond length under static equilibrium state, each bond strength (in terms of force) equals to the gradient of the potential energy along with corresponding chemical bond. Then there must be a constraint condition among each chemical bond in view of theorem (2) under the stable equilibrium.

Other possible application fields of this paper include most of the problem arisen in civil engineering and industry design such as the factory location; the city drainage system; the choice of the trifurcation point of traffic roads. In a word, the mechanical model of the static equilibrium of CPF system can solve most of the preliminary design that concerned problem of the minimal weighted distance summation. Its application can spare much manpower and material resource, decrease social operation cost and promote the economy efficiency. Meanwhile, it can work as a powerful tool to study some problems of mathematics, mechanics and even the gravitation field. 


\section{Acknowledgements}

This work was supported by the National Natural Science Foundation of China under Grant No.10775105.

\section{REFERENCES}

[1] H. William, T. S. H. George, J. Ping and C. W. L. Henry, "A Hybrid Genetic Algorithm for the Multi-depot Vehicle Routing Problem," Engineering Applications of Artificial intelligence, Vol. 21, No. 4, 2008, pp. 548-557. doi:10.1016/j.engappai.2007.06.001

[2] Benoit Crevier, Jean-François Cordeau and Gilbert Laporte, "The Multi-depot Vehicle Routing Problem with Inter-depot Routes," European Journal of Operational Research, Vol. 176, No. 4, 2007, pp. 756-773. doi:10.1016/j.ejor.2005.08.015

[3] Rubén Ruiz, Concepción Maroto and Javier Alcaraz, "A Decision Support System for a Real Vehicle Routing Problem," European Journal of Operational Research, Vol. 153, No. 3, 2004, pp. 593-606. doi:10.1016/S0377-2217(03)00265-0
[4] Bruce L.Golden, Gilbert Laporte and Éric D. Taillard, "An Adaptive Memory Heuristic for a Class of Vehicle Routing Problems with Minmax Objective," Computers \& Operations Research, Vol. 24, No. 5, 1997, pp. 445-452. doi:10.1016/S0305-0548(96)00065-2

[5] Emmanouil E. Zachariadis, Christos D. Tarantilis and Chris T. Kiranoudis, "An Adaptive Memory Methodology for the Vehicle Routing Problem with Simultaneous Pick-ups and Deliveries," European Journal of Operational Research, Vol. 202, No. 2, 2002, pp. 401-411. doi:10.1016/j.ejor.2009.05.015

[6] C. D. Collinson, "Introductory Mechanics," London: Edward Arnold Ltd., Vol. 2-3, 1980, pp. 41-42.

[7] Z. X. Zhu and Q. Z. Zhou, "Theoretical Mechanics," Beijing: Beijing University Press, Vol. 292-293, 1982, pp. 313-314.

[8] G. Y. Yu and G. Q. Zhou, "Electrodynamics," Beijing: Higher Education Press, Vol. 8-9, 1997, pp. 51-52.

[9] K. David, C. Field and Wave, “Electromagnetics," New York: Addison-Wesley Publishing Company, Inc., 1983. pp. 84-85. 